\title{
Nova abordagem cirúrgica no tratamento de estrabismos incomitantes: relato de caso
}

\author{
New surgical aproach in the treatment of incomitant strabismus:case report
}

\author{
Geraldo de Barros Ribeiro ${ }^{1}$ \\ Henderson Celestino de Almeida ${ }^{2}$ \\ Eduardo Moritz dos Santos ${ }^{3}$
}

Trabalho realizado no Hospital São Geraldo da Universidade Federal de Minas Gerais - UFMG.

${ }^{1}$ Doutor em Medicina. Professor voluntário do serviço de estrabismo do Hospital São Geraldo da Universidade Federal de Minas Gerais - UFMG - Belo Horizonte (MG) - Brasil.

${ }^{2}$ Professor Titular e chefe do serviço de Estrabismo do Hospital São Geraldo da UFMG - Belo Horizonte (MG) - Brasil.

${ }^{3}$ Estagiário do serviço de Estrabismo do Hospital São Geraldo da UFMG - Belo Horizonte (MG) - Brasil.

Endereço para correspondência: Geraldo de Barros Ribeiro. Av. Silviano Brandão, 1600 - Belo Horizonte (MG) CEP 31015-000

E-mail: gribeiro@br.inter.net

Recebido para publicação em 04.04.2005

Versão revisada recebida em 13.12.2005

Aprovação em 30.01.2006

Nota Editorial: Depois de concluída a análise do artigo sob sigilo editorial e com a anuência do Dr. Carlos Ramos Souza-Dias sobre a divulgação de seu nome como revisor, agradecemos sua participação neste processo.

\section{RESUMO}

Os autores relatam a utilização da técnica cirúrgica proposta por Scott, em 1994, de recuo e ressecção do mesmo músculo extra-ocular no tratamento de estrabismo incomitante horizontal. A paciente, do sexo feminino, 22 anos, apresentava estrabismo horizontal incomitante, com uma esotropia de $9^{\Delta}$ para longe e $30^{\Delta}$ para perto. Submetida a recuo e ressecção do músculo reto medial direito, apresentou resultados satisfatórios a longo prazo, mostrando que esta técnica pode fazer parte do arsenal terapêutico para correção de estrabismos incomitantes.

Descritores: Esotropia/cirurgia; Movimentos oculares; Músculos oculomotores/cirurgia; Procedimentos cirúrgicos oftalmológicos; Relatos de casos [tipo de publicação]

\section{INTRODUÇÃO}

O tratamento dos estrabismos incomitantes continua sendo um desafio aos estrabólogos. As principais causas de desvios horizontais incomitantes são as paresias musculares, restrições oculares, divergência horizontal dissociada, relação convergência acomodativa/acomodação (CA/A) alta ou cirurgias prévias ${ }^{(1)}$.

Diversos tratamentos já foram propostos, como simples recuos ou ressecções, que produziram apenas razoável alinhamento em posição primária do olhar (PPO), hipocorreção no campo de ação do músculo afetado e hipercorreção no campo oposto, e a cirurgia bilateral assimétrica, porém nem sempre com resultados satisfatórios ${ }^{(2)}$.

Cüppers propôs em 1976 a mioescleropexia retroequatorial (fadenoperation $)^{(3)}$. A técnica cirúrgica consiste da sutura do músculo reto à esclera, posterior à inserção anatômica, criando assim uma segunda inserção. Em princípio, esta segunda inserção irá diminuir o efeito da contração na rotação do bulbo ocular, sem alterar o equilíbrio entre agonista e antagonista em posição primária do olhar ${ }^{(3-4)}$. A partir de então, essa técnica tem sido muito utilizada para tratamento de desvios incomitantes. Tem também sido indicada para tratamento da divergência vertical dissociada (DVD), de esotropia com relação CA/A alta e síndrome de bloqueio do nistagmo ${ }^{(3,5)}$. Apesar de apresentar resultados satisfatórios, essa técnica é de difícil execução e pode envolver riscos ${ }^{(1)}$. Particularmente perigosa é a realização deste procedimento no reto lateral, pois a inserção do músculo oblíquo inferior está no campo da cirurgia e as suturas acabam sendo feitas abaixo ou próximas à mácula ${ }^{(2)}$. Outras complicações foram relatadas, como midríase persistente, atrofia óptica, edema macular, hemorragia vítrea e descolamento de coróide $^{(3)}$. Reoperações tornam-se mais complexas devido à fibrose do músculo anteriormente à sutura de fixação posterior ${ }^{(2)}$.

Scott, em 1994, descreve uma nova técnica, na qual se cria o efeito da 
fixação posterior, sem a colocação de $\operatorname{suturas}^{(6)}$. Nesta técnica, uma porção do músculo é ressecada e o mesmo músculo é recuado. Este recuo deve ser maior ou igual à ressecção, para poder produzir uma diminuição seletiva da função do músculo em seu campo de ação ${ }^{(2)}$ (Figura 1).

O objetivo deste relato de caso é apresentar o resultado obtido com o uso desta técnica proposta por Scott, para tratamento de estrabismo horizontal incomitante.

\section{RELATO DE CASO}

LJIS, 22 anos, solteira, recepcionista, natural de Belo Horizonte, procurou atendimento oftalmológico no consultório de um dos autores em 11/09/01, queixando-se de desvio ocular principalmente para perto e insatisfeita com a estética.

Relatou história de estrabismo desde os 3 anos. Usou oclusor para tratamento da ambliopia dos 3 aos 7 anos. Cirurgia prévia de estrabismo há 6 anos, com recuo de $4 \mathrm{~mm}$ do reto

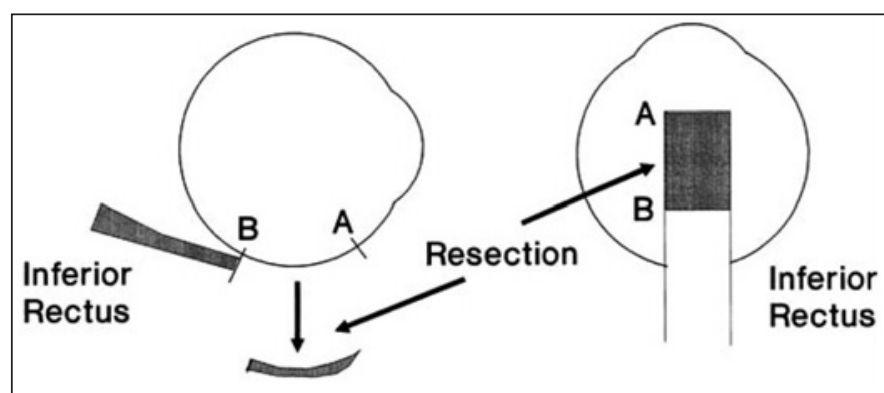

Fonte: Bock CJ Jr, Buckley EG, Freedman SF. Combined resection and recession of a single rectus muscle for the treatment of incomitant strabismus. J AAPOS. 1999;3(5):263-8(2).

Figura 1 - Recuo e ressecção de um mesmo músculo extra-ocular. Uma porção do músculo é ressecada e o músculo é inserido posteriormente (B). medial esquerdo (RME) e ressecção de $6 \mathrm{~mm}$ do reto lateral esquerdo (RLE) com deslocamento superior das inserções de $2 \mathrm{~mm}$. Foi submetida também a uma aplicação de 5 unidades de toxina botulínica há 5 anos no reto medial direito. Não apresentava alterações sistêmicas.

Ao exame oftalmológico, apresentava acuidade visual sem correção de 1,0 no olho direito e de 0,8 no olho esquerdo, a qual não melhorava com correção. Biomicroscopia sem alterações. Ao exame de fundo-de-olho apresentava cicatrizes de retinocoroidite nas arcadas nasal e temporal inferiores em OE. Refração estática de +0,50 -0,50 a 90 em ambos os olhos (AO).

$\mathrm{O}$ "cover" teste alternado revelou esotropia (ET) de $9^{\Delta}$ para longe e de $30^{\Delta}$ para perto. Em supraversão, a 6 metros, assim como em infraversão, a ET era de $9^{\Delta}$. Apresentava discreta limitação na adução do olho esquerdo (-1/-4) (Figura 2).

A paciente foi submetida à correção cirúrgica do estrabismo em 17/04/02, sendo realizada ressecção de $4 \mathrm{~mm}$ com recuo de $6 \mathrm{~mm}$ do reto medial direito (RMD).

No $1^{\circ}$ dia pós-operatório (PO), apresentava para longe ET $4^{\Delta}$ e para perto ET' $14^{\Delta}$, com discreta hipofunção do RMD (-1,5/-4).

No $7^{\circ} \mathrm{PO}$, encontrou-se ET $3^{\Delta}$ para longe e um ET' $12^{\Delta}$ para perto, com leve melhora na hipofunção do RMD (-1/-4).

Apresentava, ao exame no $15^{\circ} \mathrm{PO}$, ET para longe de $3^{\Delta} \mathrm{e}$ para perto de $12^{\Delta}$, mantendo a discreta hipofunção de RMD $(-0,5 /-4)$ (Figura 3).

No $30^{\circ}$ dia PO, permanecia com ET $2^{\Delta}$ para longe e $12^{\Delta}$ para perto, mantendo também a discreta hipofunção do RMD. A paciente ficou satisfeita com resultado cirúrgico.

A paciente manteve-se com quadro estável por mais de um ano, tendo perdido contato no ano seguinte, alegando estar satisfeita com o resultado da cirurgia. No PO tardio de 2 anos, apresentava ET $5^{\Delta}$ para longe e $18^{\Delta}$ para perto. Apresentava, às versões, apenas discreta hipofunção dos retos mediais $(-0,5 /-4)$ (Figura 4).
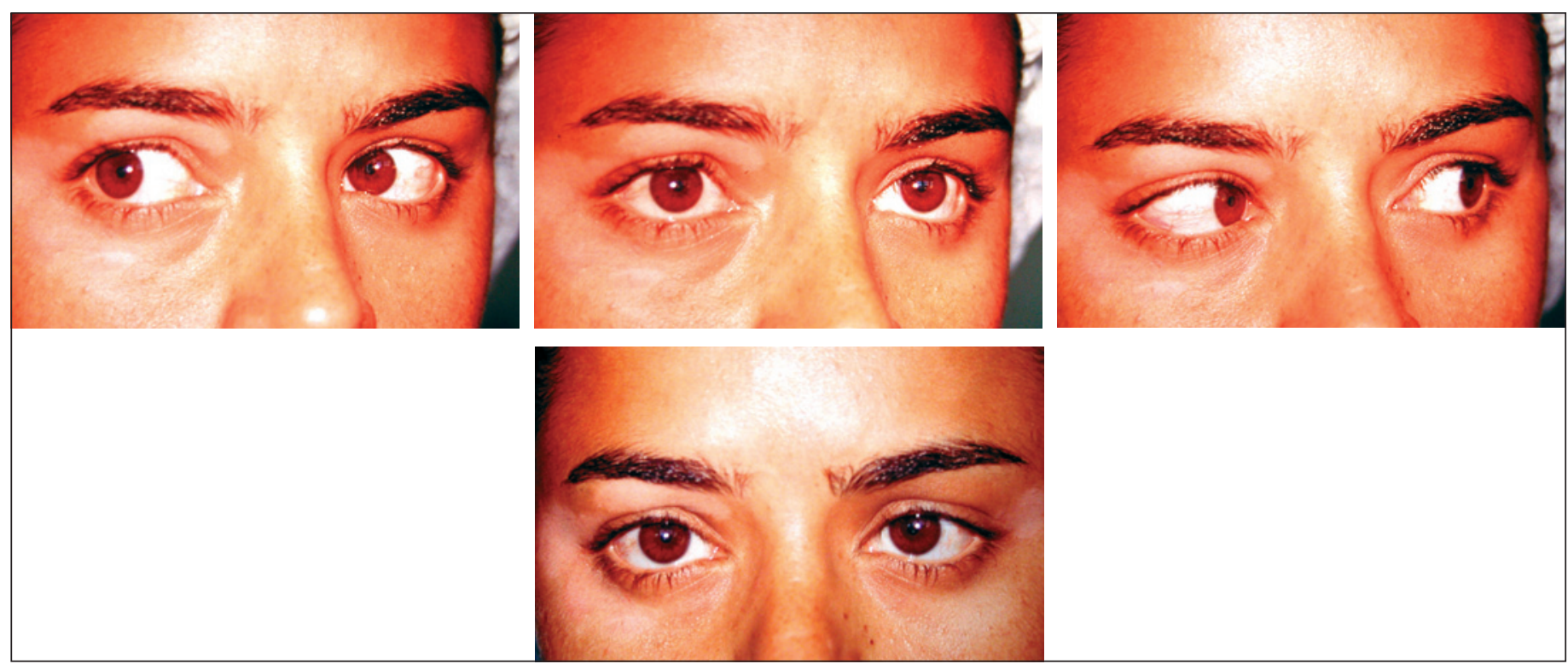

Figura 2 - Paciente no período pré-operatório. ET de $9^{\Delta}$ e ET' de $30^{\Delta}$, com discreta limitação de adução reto medial esquerdo. 


\section{DISCUSSÃO}

Os estrabismos incomitantes geralmente são um desafio ao estrabólogo. O uso da técnica de recuo e ressecção do mesmo músculo, proposta por Scott em 1994, pode ser, em casos selecionados, uma boa opção para o tratamento destes tipos de estrabismo. Ela visa enfraquecer o músculo em seu campo de ação sem que haja alterações significativas na posição primária do olhar ${ }^{(2,6)}$.

A paciente submetida a este procedimento apresentava estrabismo horizontal incomitante, mesmo tendo sido submetida a cirurgia prévia para correção de esotropia.
Alguns autores em 1999, utilizando a mesma técnica proposta por Scott para tratamento de estrabismos incomitantes, operaram 12 pacientes, obtendo redução significativa da incomitância tanto para os estrabismos verticais quanto para os horizontais $^{(2)}$.

A proposta inicial de Scott era a de uma grande ressecção, porém concordamos com a modificação, feita por Bock et al., de diminuir a porção ressecada do músculo, pela impossibilidade de prever os efeitos a longo prazo $^{(2)}$.

Apesar do efeito promissor, alguns detalhes devem ser analisados, como a sua estabilidade e as possíveis reoperações em longo prazo.
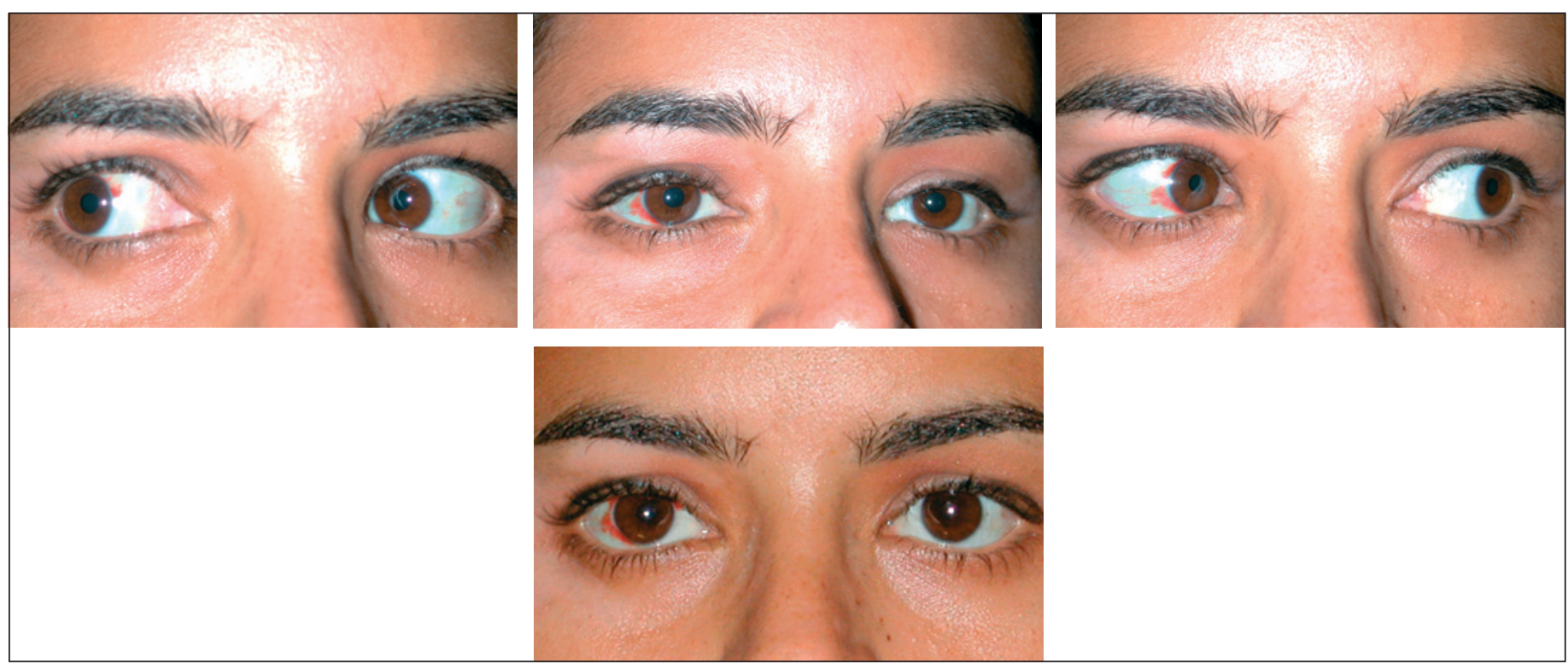

Figura 3 - Paciente no 15 PO. ET $3^{\Delta}$ e ET'12 ${ }^{\Delta}$, com discreta hipofunção de RMD e RME.
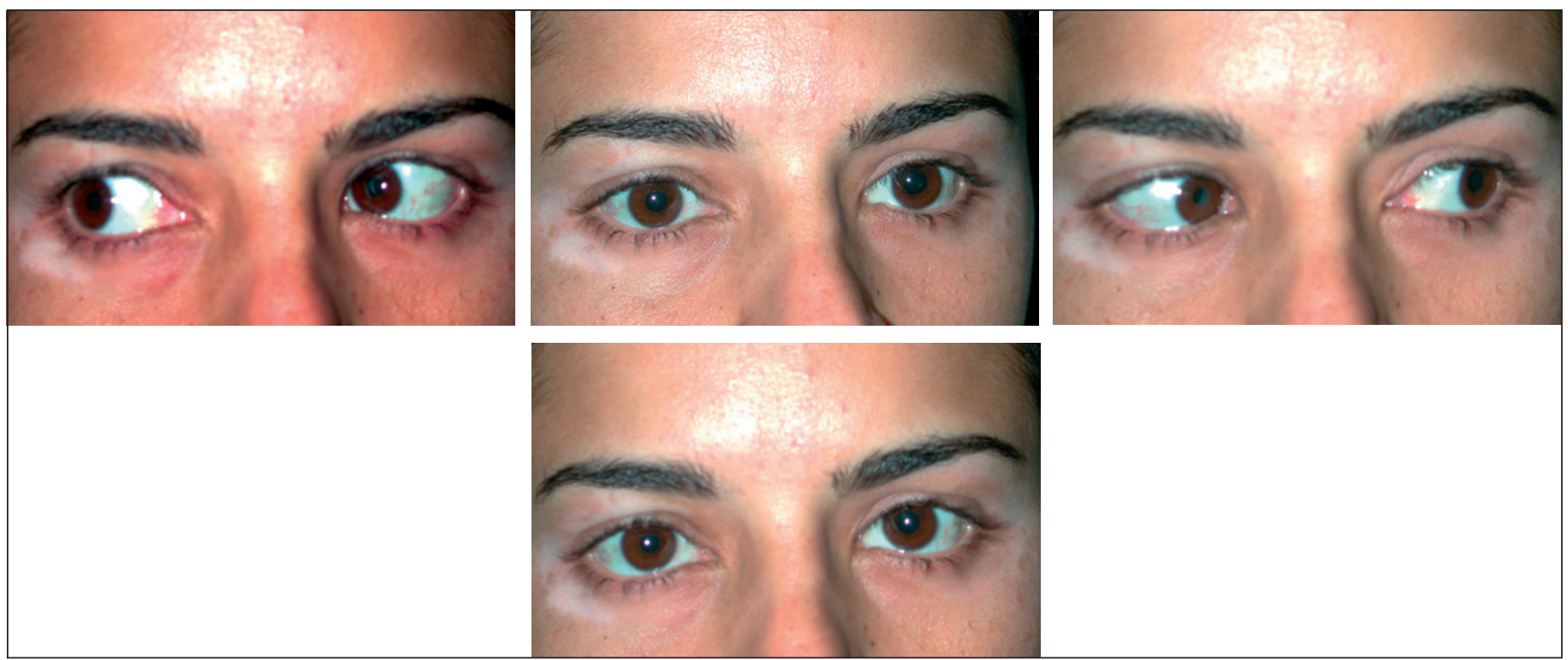

Figura 4 - Paciente com 2 anos após a operação. Apresentava ET $5^{\Delta}$ e ET'18 ${ }^{\Delta}$, com discreta limitação do RMD e RME. 
Bock et al., concluíram que este é um procedimento seguro para estrabismos incomitantes em que os retos laterais estejam envolvidos, possibilitando também a utilização de suturas ajustáveis para eliminar o problema da fixação posterior, porém, para os outros músculos, concluiu não ter experiência suficiente para julgar a eficácia do procedimento $^{(2)}$.

Em nosso caso, a cirurgia mostrou-se eficaz, reduzindo o desvio de forma significativa, sendo o resultado encontrado após dois anos satisfatório tanto para o cirurgião quanto para a paciente.

Apesar do bom resultado inicial encontrado, entendemos ser necessária a realização da técnica descrita em maior número de casos para que se comprove a sua eficácia em outros músculos e, também, estabelecermos uma relação confiável entre recuo-ressecção.

\section{ABSTRACT}

The authors report the utilization of the surgical technique proposed by Scott, in 1994, of recess and resection of the same extraocular muscle in the treatment of incomitant horizontal strabismus. A 22-year-old female presented with incomitant strabismus, with an esotropia of $9^{\Delta}$ for distant and $30^{\Delta}$ for near. She was submitted to a recess and resection of the right medial rectus. She presented satisfactory results in the long-term, showing that this technique could be part of the therapeutic arsenal for correction of incomitant strabismus.

Keywords: Exotropia/surgery; Eye movements; Oculomotor muscles/surgery; Ophthalmologic surgical procedures; Case reports [publication type]

\section{REFERÊNCIAS}

1. Souza-Dias C, Prieto-Diaz J. Cirurgia do estrabismo. In: Prieto-Diaz J, SouzaDias C. Estrabismo. 4a ed. São Paulo: Santos; 2002. p.475-7.

2. Bock CJ Jr, Buckley EG, Freedman SF. Combined resection and recession of a single rectus muscle for the treatment of incomitant strabismus. J AAPOS. 1999;3(5):263-8.

3. Cüppers C. The so-called "fadenoperation" (surgical considerations by well defined changes of the arc of contact). In: Fells P, editor. Transactions of the second congress of International Strabismological Association. Marseilles (France): Diffusion Generale de Librairie; 1976. p.395-400.

4. Millicent M, Peterseim W, Buckley EG. Medial rectus fadenoperation for esotropia only at near fixation. J AAPOS. 1997;1(3):129-33.

5 . Von Noorden GK. Indications of the posterior fixation operation in strabismus. Ophthalmology. 1978;85(5):512-20.

6. Scott AB. Posterior fixation: adjustable and without posterior sutures. In: Lennerstrand G, editor. Update on strabismus and pediatric ophthalmology: Proceedings of the june 1994 Joint ISA and AAPO\&S Meeting. Vancouver, Canada. Boca Raton (FL): CRC; 1995. p.399.

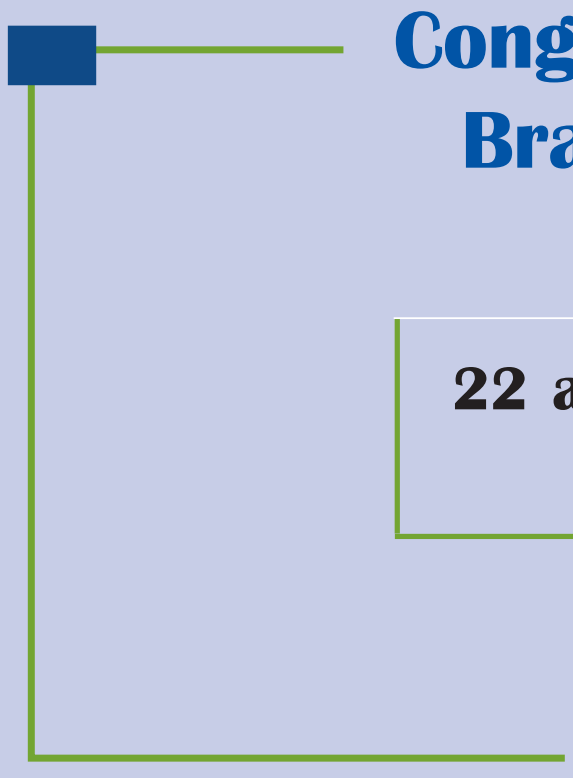

\section{INFORMAÇÕES \\ E-mail: sibi@uol.com.br}

